\title{
Virtual Agent Representation for Critical Transactions
}

\author{
Ningyuan Sun \\ University of Luxembourg \\ 4364 Esch-sur-Alzette, Luxembourg \\ ningyuan.sun@uni.lu
}

\author{
Jean Botev \\ University of Luxembourg \\ 4364 Esch-sur-Alzette, Luxembourg \\ jean.botev@uni.lu
}

\begin{abstract}
With their proliferation, virtual agents become increasingly involved in critical transactions between groups and individuals. Mediated-reality technologies such as virtual and augmented reality present both new opportunities and challenges for representing such agents. This study explores which agent representation users prefer for critical transactions such as a substantial financial investment. We compare our experimental results from a more general group of users with those from a control group of savvy users familiar with virtual environments and computer games, deriving initial design recommendations and an outlook on further research. Generally, a human or humanoid representation is deemed preferable, while, among the alternatives, object representations are favored over zoomorphic, plant-based, or purely symbolic options.
\end{abstract}

\section{CCS CONCEPTS}

- Human-centered computing $\rightarrow$ Virtual reality; Mixed / augmented reality; • Computing methodologies $\rightarrow$ Intelligent agents; Appearance and texture representations.

\section{KEYWORDS}

Agent representation, user-agent interaction, critical transactions

\section{ACM Reference Format:}

Ningyuan Sun and Jean Botev. 2021. Virtual Agent Representation for Critical Transactions. In International Workshop on Immersive Mixed and Virtual Environment Systems (MMVE '21), September 28-October 1, 2021, Istanbul, Turkey. ACM, New York, NY, USA, 5 pages. https://doi.org/10.1145/3458307. 3463372

\section{INTRODUCTION}

Agent technology has rapidly evolved in recent decades, and virtual agents are now omnipresent in a broad spectrum of human activities including e-commerce [11], social media [10], manufacturing [20], and many more. With the proliferation of mobile Internet-enabled devices and the Internet of Things, interaction with virtual agents has become commonplace since these technologies have significantly facilitated the deployment of and access to virtual agents.

Permission to make digital or hard copies of all or part of this work for personal or classroom use is granted without fee provided that copies are not made or distributed for profit or commercial advantage and that copies bear this notice and the full citation on the first page. Copyrights for components of this work owned by others than the author(s) must be honored. Abstracting with credit is permitted. To copy otherwise, or republish, to post on servers or to redistribute to lists, requires prior specific permission and/or a fee. Request permissions from permissions@acm.org.

MMVE '21, September 28-October 1, 2021, Istanbul, Turkey

(c) 2021 Copyright held by the owner/author(s). Publication rights licensed to ACM ACM ISBN 978-1-4503-8436-0/21/09..\$15.00

https://doi.org/10.1145/3458307.3463372
The exponential growth in computing power and the upsurge of artificial intelligence research further increase the capabilities of virtual agents, which consequently play increasingly important and crucial roles [18] in our daily transactions.

This entails a growing research interest in the various aspects of virtual agent design, with representation aspects playing a significant role. Generally, having a representation is advantageous to virtual agents, and it has been shown to improve social presence [3] and spatial presence [13], the establishment of positive impressions [19], the perception of agent credibility [2], the generation of affective influence [1], and the overall user experience [13]. However, it can also have an adverse impact on user-agent interaction, e.g., in the so-called Uncanny Valley issue [12], or lead to a reduced trustworthiness of virtual agents [7].

From an overarching perspective, virtual agent representation refers to how humans can perceive such agents. It is often considered synonymous with virtual agents' visual appearance but, in fact, also involves all other sensory channels. Current virtual agents are predominantly represented by a combination of visual and auditory information. Specifically, the visual representation is often designed to convey the bulk of information since it is one of the most important cues for users to evaluate agent behavior [9].

One extreme is virtual agents without a representation, in which case users can only infer their existence. The other extreme is a multimodal representation of virtual agents through cutting-edge immersive technology, such as virtual reality (VR) or augmented reality (AR). Here, designers can combine various channels, increase the informational bandwidth, and make the interaction more immersive [5] and natural [6] compared to standard two-dimensional display technologies. However, due to the novelty and recency of mediated-reality technologies, only a relatively small amount of research has been conducted to explore the influence of agent representation specific to VR/AR environments.

A complicating factor is that there is a virtually infinite number of possible agent representations, which gives designers great liberties in their work. However, in terms of research, this leads to limited comparability of virtual agent representations and a lack of accurate metrics that precisely capture the differences between any two representations. Consequently, navigating the massive space of agent representation can be difficult. Most of the time, to distinguish between virtual agents' different representations, either a natural language description or technical details are being used. In many cases, this suffices for general discussion, but eventually, a more systematic approach to virtual agent representation will be necessary to make further research progress.

This paper constitutes the first step towards a formalization of agent representations for critical transactions in virtual environments. Before elaborating on the results in Section 3, we discuss the experimental design and test group composition in Section 2. 


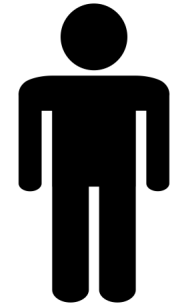

Human

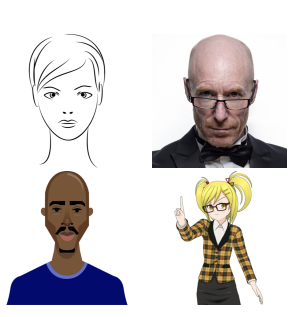

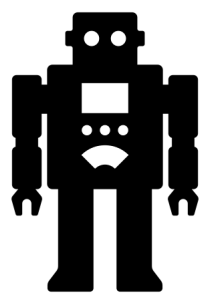

Humanoid

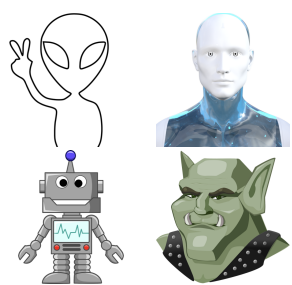

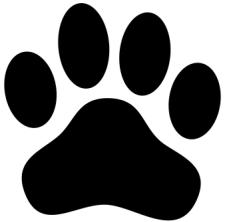

Organic Non-Human

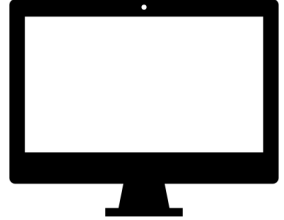

Inanimate Object
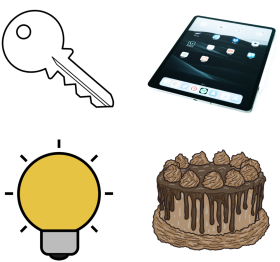

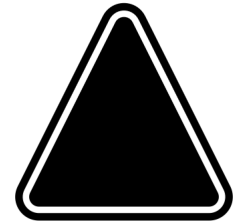

Symbol
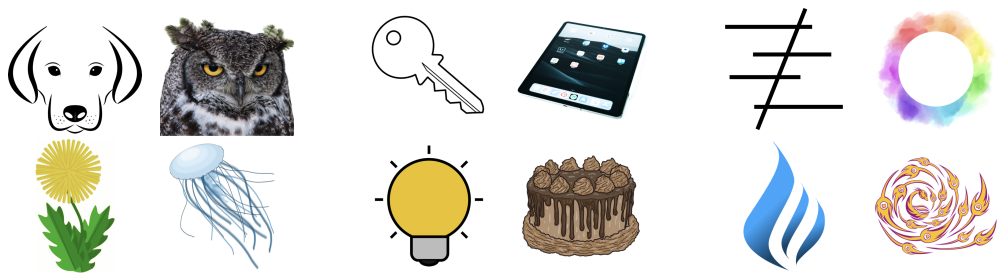

Figure 1: Virtual agent representation categories with example instances.

We conclude the paper in Section 4 with a set of initial design recommendations and an outlook on further research.

\section{EXPERIMENTAL DESIGN}

We conducted an experiment to investigate users' general preferences for virtual agent representation in the context of critical transactions. The five categories offered to the participants distinguish the grade of human semblance, with possible representations ranging from human, over humanoid, organic non-human (i.e., zoomorphic or plant-based), and inanimate objects (i.e., items or devices), to purely symbolic. Figure 1 shows the illustrated categories, each accompanied by four specific examples below its name. The examples show different potential instances of the respective category as line drawing (top left), photorealistic image (top right), and stylized as symbolic low-detail drawing (bottom left) or descriptive high-detail drawing (bottom right).

The degree of human likeness is a common criterion in studies on agents' appearance, and most representation design -in both academic and industrial contexts- falls into one of these categories. However, categorizing representation is a complex task as a virtual agent can essentially be represented in any shape or form. Depending on the research focus, categorizations might change. In [15], for instance, species and realism are identified as the key variables of agent appearance. The research indicates different species (human, animal, robots, objects, and mystical creatures) and realism levels (stylization, resolution, and detailedness) which, in later work by the same authors, was demonstrated to have an impact on older people's willingness to interact with an agent $[16,17]$.

Our categorization comprises all those factors and further adds non-zoomorphic species, as well as the often neglected category of symbolic representations. Due to their clarity and comprehensibility, the latter potentially constitute helpful and immediate indicators in critical transactions. For each category, the examples involve diverse visual fidelities to give the participants a sense of the difference.

\subsection{Experiment Setup}

For experimenting with different groups of participants (cf. Section 2.2), we used the Amazon Mechanical Turk ${ }^{1}$ crowdsourcing platform. The interactive survey is identical in layout and content to Figure 1; however, unlike the level of human semblance decreasing from left to right as in the figure, the order of the categories is randomized for each participant. For instance, one participant indeed may see the sequence human, humanoid, organic non-human, inanimate object, and symbol, while another participant may be presented with the sequence humanoid, human, inanimate object, symbol, and organic non-human. This rearrangement avoids potential bias caused by a fixed order.

Each participant is given the following short scenario brief to contextualize the scenario as broadly as possible:

"Imagine you have a large amount of money to invest through a virtual agent."

This description addresses a critical transaction and universal issue independent of, for instance, the geographic region or economic situation. The participants are asked to rank the categories uniquely from 1-5, i.e., their favorite option is ranked 1 (best), while their least preferable option is assigned rank 5 (worst). This ranking mode allows for a fine-grained data analysis, in particular related to alternative options.

\subsection{Group Composition}

Using a global crowdsourcing platform permits reaching a broad audience spanning many different demographic factors such as age or profession, which allows for a more generalized study compared to merely employing university students.

We recruited a total of 100 MTurk Master Workers in order to gather statistically significant results. Master workers have a proven track record of successfully and diligently performing a large number of different tasks on the platform. Among those, 85

\footnotetext{
${ }^{1}$ https://www.mturk.com
} 
participants form the test group without restrictions in terms of their background, while the rest was filtered according to whether they had already purchased video games online. These 15 participants serve as a control group to determine whether there is a measurable difference between less involved and more savvy users familiar with virtual environments and computer games. In such settings, virtual agents are ubiquitous. Users familiar with game-based virtual environments, therefore, likely have already interacted with or experienced various agent types. Consequently, it is interesting to explore the preference landscape of savvy users compared to that of a more general audience.

\section{RESULTS}

This section discusses the empirical data obtained from the interactive survey, providing a general analysis of the results in Section 3.1, followed by a detailed comparison with the results for the control group of savvy users in Section 3.2.

Table 1 contains the raw data of the survey results from a total of 100 participants (85 test group, 15 control group). The unique representation category ranking follows a 5-point scale comprising 1 (best), 2 (better), 3 (neutral), 4 (worse), and 5 (worst).

Table 1: Survey responses.

\begin{tabular}{|c|r||c|c|c|c|c||}
\cline { 3 - 7 } \multicolumn{2}{c|}{} & \multicolumn{6}{|c|}{ Ranking } \\
\hline Category & Group & 1 & 2 & 3 & 4 & 5 \\
\hline \hline \multirow{2}{*}{ Human } & Test & 47 & 6 & 5 & 8 & 19 \\
\cline { 2 - 7 } & Control & 14 & 0 & 1 & 0 & 0 \\
\hline \hline \multirow{2}{*}{ Humanoid } & Test & 9 & 39 & 16 & 16 & 5 \\
\cline { 2 - 7 } & Control & 0 & 9 & 3 & 3 & 0 \\
\hline \hline \multirow{2}{*}{ Org. Non-Human } & Test & 10 & 9 & 21 & 21 & 24 \\
\cline { 2 - 7 } & Control & 0 & 0 & 3 & 9 & 3 \\
\hline \hline \multirow{2}{*}{ Inan. Object } & Test & 8 & 17 & 27 & 16 & 17 \\
\cline { 2 - 7 } & Control & 1 & 5 & 5 & 1 & 3 \\
\hline \hline \multirow{2}{*}{ Symbol } & Test & 11 & 14 & 16 & 24 & 20 \\
\cline { 2 - 7 } & Control & 0 & 1 & 3 & 2 & 9 \\
\hline
\end{tabular}

As the aggregated score in Figure 2 indicates, human and humanoid representations overall are clearly preferred. Both the Friedman test $\left(Q=30.824, p=3.3 \times 10^{-6}\right)$ and post-hoc analysis via Nemenyi test (cf. Table 2) corroborate this observation.

Table 2: Nemenyi test p-values.

\begin{tabular}{|c|c|c|c|c|c|}
\hline Category & Human & Humanoid & Org. N.-H. & Inan. Obj. & Symbol \\
\hline Human & 1.000 & 0.772 & 0.001 & 0.005 & 0.001 \\
\hline Humanoid & 0.772 & 1.000 & 0.005 & 0.136 & 0.034 \\
\hline Org. N.-H. & 0.001 & 0.005 & 1.000 & 0.772 & 0.900 \\
\hline Inan. Obj. & 0.005 & 0.136 & 0.772 & 1.000 & 0.900 \\
\hline Symbol & 0.001 & 0.034 & 0.900 & 0.900 & 1.000 \\
\hline
\end{tabular}

Of the alternatives, object representation is favored over nonhuman, i.e., zoomorphic or plant-based, and symbolic representations. However, the difference between the alternatives is less evident and will be discussed in more detail in the following.

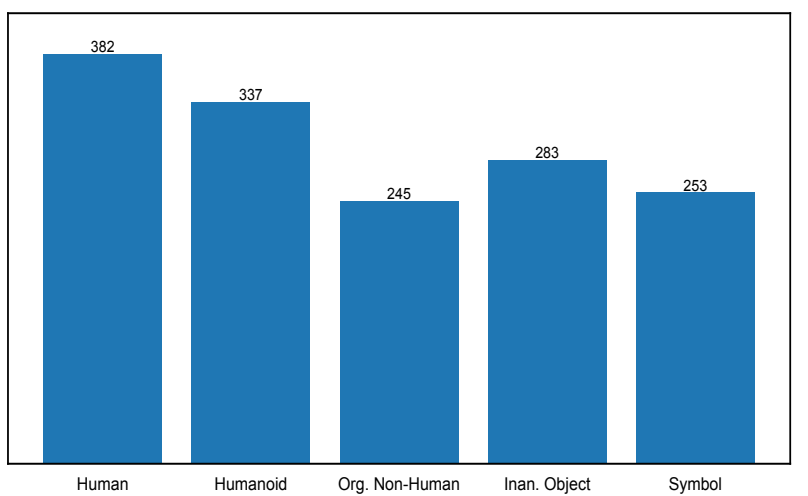

Figure 2: Aggregate results, inverse scale rank sum.

\subsection{General Analysis}

As Figure 3 shows, a human virtual agent representation in the context of critical transactions is clearly favored across groups. In the test group, around $63 \%$ consider a human representation to be better or best, while the results of the control group constitute a nearly unanimous vote with more than $93 \%$ preferring a human virtual agent representation. The result is in line with findings showing that anthropomorphic features generally facilitate humanagent interaction [8].

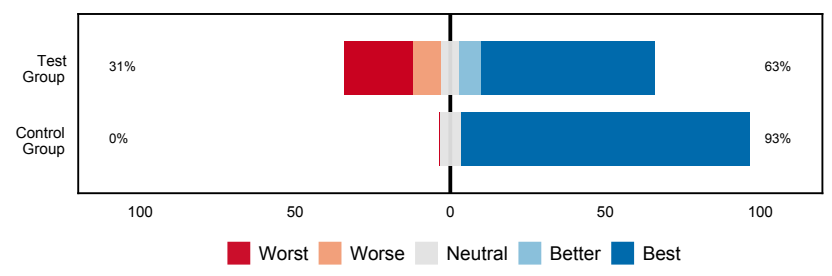

Figure 3: Results, human representation.

A humanoid virtual agent representation, i.e., a non-human being but with characteristics resembling those of a human, is the similarly unequivocal second for scenarios involving critical transactions. As shown in Figure 4, almost half (46\%) of the test group and more than $60 \%$ of the control group voted the humanoid option second.

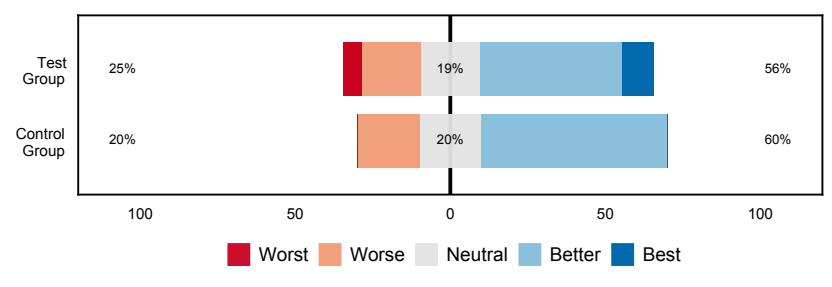

Figure 4: Results, humanoid representation.

As each participant is presented with a randomized sequence of the categories, the tendency towards strong human semblance is 
particularly notable. This is further supported by a strong correlation between the positive rankings of those two categories; among the 47 participants who picked a human representation as their favorite, 33 chose a humanoid representation as their second option. A humanoid representation is therefore an excellent alternative when considering critical transactions with virtual agents. In fact, it is not uncommon that a human representation is unfeasible or undesirable, especially in light of associated issues such as the Uncanny Valley. Designers who strive for photorealistic virtual agents may also refrain from using a fully human representation due to the high complexity of animations and computational cost.

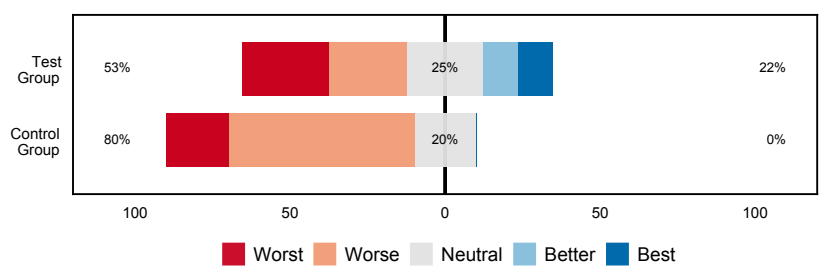

Figure 5: Results, organic non-human representation.

The organic non-human virtual agent representation category is on the other end of the spectrum, ranked negatively across groups. As shown in Figure 5, more than half of the test group (53\%) and about $80 \%$ of the control group ranked this category, i.e., zoomorphic or plant-based representations of virtual agents, as worse or worst.

This is interesting since zoomorphic agents have been considered useful in the past [14], and many projects involved such a representation, e.g., Microsoft's canine Office suite assistant Rocky.

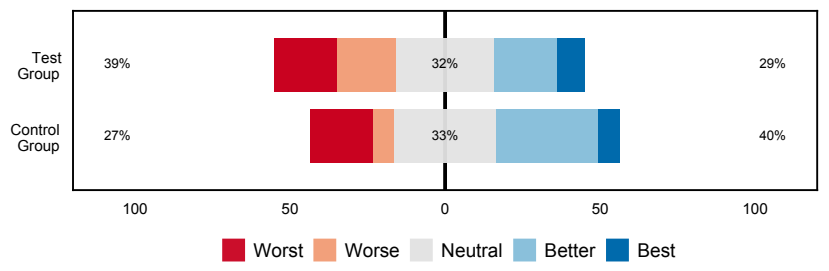

Figure 6: Results, inanimate object representation.

Object representations, as shown in Figure 6, are favored over the other non-human alternatives, i.e., the zoomorphic, plant-based, or purely symbolic options. Also here, the tendency within the test group was affirmed by the control group. Generally, representing virtual agents with inanimate objects is closest to the experience horizon of the average user that accesses services via desktop computers, mobile devices or other items such as smart speakers.

Finally, the symbolic representation of virtual agents in critical agents received similarly negative results as the organic non-human representation category. $52 \%$ of the test group and $73 \%$ of the control group ranked it worse or worst, as shown in Figure 7. An interesting detail here is the pronounced dislike for this category among the more savvy users in the control group (cf. Section 3.2). Overall, the seemingly low information bandwidth and limited interactivity does not make users consider a symbolic representation a viable option in critical transaction contexts.

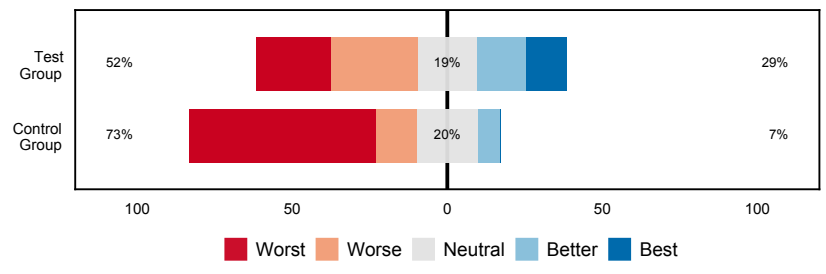

Figure 7: Results, symbolic representation.

From an overarching perspective, the clear polarization of the participants with regards to human-like representation is noteworthy. The majority of the participants $(78 \%$ of the test group and $93 \%$ of the control group) rank the human representation either best or worst, which might be because people are more experienced in evaluating the interaction with another human rather than with non-human beings or entities. The general inclination towards human likeness in scenarios involving critical transactions is, for instance, also reflected by the majority of online agents in e-commerce being represented as human or humanoid [4].

Based on this consideration, the general dislike for the organic non-human representation might not only be attributed to low information bandwidth and limited interactivity but merely to the minimal degree of human semblance. In the test group, 35 out of 47 participants ranking the human representation best have also given negative rankings to the organic non-human representation. On the other hand, zoomorphic and plant-based options might adversely impact the participants' assessment, explaining that the inanimate object and symbolic representation categories, despite clearly distinct from humans, are more popular in comparison.

\subsection{Control Group}

While similar in the overall outcome, the results shown in Figures 3 to 7 in Section 3.1 indicate more pronounced preferences on the control group side. There is an almost unanimous ranking of a human virtual agent representation as best (93\%), and a more moderate but still strong preference for a humanoid representation which $60 \%$ of the control group ranked second (better). This predilection for human semblance may stem from the frequent occurrence of human-like avatars and non-player characters (NPCs) in computer games, which users engaging in such virtual environments are particularly familiar with.

On the other end of the spectrum, the dislike in particular symbolic representations is much more pronounced. Such virtual agent representations are ranked worst by more than $60 \%$ of the control group. While still far from a preferred option, organic non-human representations are less loathed than in the test group. This is possibly also due to the experience with game-based virtual environments, where agents with zoomorphic or plant-based representations are not uncommon, e.g., a wolf blacksmith, a chicken courier, or a tree oracle. In turn, a purely symbolic agent representation is relatively rare in computer games and may explain why of all the categories, this representation category was by far perceived as least desirable for critical transactions and ranked worst or worse by almost $73 \%$ of the control group but only around half (52\%) of the test group. 


\section{CONCLUSION AND OUTLOOK}

In this study, we conducted a survey-based experiment to determine user preferences regarding virtual agent representation in the context of critical transactions in mediated-reality settings. The results indicate a clear dividing line between human-like representations (human or humanoid) and non-human representation variants (organic non-human, inanimate object, or symbolic). A human or humanoid representation of virtual agents is generally well accepted and -provided that known interaction issues such as the Uncanny Valley are avoided- thus qualifies for application in critical transactions. The preference for the remaining categories is more diffuse; however, if a human-like representation is not possible or desired, object representations appear generally favorable over zoomorphic or purely symbolic representations. More savvy users familiar with virtual environments and computer games, show an even clearer preference towards human representations. However, likely due to their experience, they show a less pronounced dislike of zoomorphic or plant-based virtual agent representations.

Albeit designed to integrate a wide range of established representations, the categorization employed in this research constitutes a potential limitation. The five categories - human, humanoid, organic non-human, inanimate object, and symbol- may not unequivocally capture certain representation types. For instance, an anthropomorphic object representation is difficult to classify into any of the five categories but would fall somewhere between the organic non-human and inanimate object categories. Besides, the individual categories are determined based on the agent representations' human semblance. This semblance includes different aspects such as anthropomorphism, perceived consciousness, intelligence, and more; it is, therefore, difficult to determine the extent to which each aspect accounts for the users' preferences. Dynamic agent representation, i.e., agent behaviors or animations, also plays a critical role. The same agent representation might deliver different messages, such as an equal human representation performing different gestures. Conversely, the same behavior or animation may hold a different meaning when applied to another agent representation. For example, a crying baby might signal something other to the user than a crying adult. Finally, virtual environments are not bound to many of the restrictions a real environment imposes on agent design. As a consequence, there is an endless number of possible agent representations, which complicates their classification. Further efforts by the research community are needed to fully capture relevant agent representations and which types are most common in specific scenarios.

Mediated-reality technologies and virtual agents are playing increasingly important roles in our daily lives. This study provides initial guidance to developers and researchers on virtual agent representation in light of critical transactions. We plan to gather further empirical evidence from more extensive test groups and finer subcategories to facilitate such agents' design based on the presented results. Particularly the relationship between human representation and critical transaction decisions can be very nuanced and opens up many further lines of inquiry.

\section{ACKNOWLEDGMENTS}

This study has been supported by the Luxembourg National Research Fund (FNR) under grant number 12635165.

\section{REFERENCES}

[1] Amy L Baylor and Rinat B Rosenberg-Kima. 2006. Interface Agents to Alleviate Online Frustration. In Proceedings of the 2006 International Conference of the Learning Sciences, Vol. 1. 30-36.

[2] Amy L Baylor and Jeeheon Ryu. 2003. The Effects of Image and Animation in Enhancing Pedagogical Agent Persona. Journal of Educational Computing Research 28, 4 (2003), 373-394.

[3] Frank Biocca and Mark R Levy. 2013. Communication in the Age of Virtual Reality. Routledge.

[4] Veena Chattaraman, Wi-Suk Kwon, Juan E Gilbert, and Soo In Shim. 2011. Virtual Agents in E-Commerce: Representational Characteristics for Seniors. Journal of Research in Interactive Marketing 5, 4 (2011), 276-297.

[5] Daniel Cliburn, Stacy Rilea, Justin Charette, Ross Bennett, Daniel Fedor-Thurman, Todd Heino, and David Parsons. 2009. Evaluating Presence in Low-Cost Virtual Reality Display Systems for Undergraduate Education. Journal of Computing Sciences in Colleges 25, 2 (2009), 31-38.

[6] Riccardo Galdieri and Marcello Carrozzino. 2018. Natural Interaction in Virtual Reality for Cultural Heritage. In Proceedings of the 2018 International Conference on VR Technologies in Cultural Heritage. 122-131.

[7] Li Gong and Clifford Nass. 2007. When a Talking-Face Computer Agent Is Half-Human and Half-Humanoid: Human Identity and Consistency Preference. Human Communication Research 33, 2 (2007), 163-193.

[8] Agneta Gulz and Magnus Haake. 2006. Visual Design of Virtual Pedagogical Agents: Naturalism Versus Stylization in Static Appearance. In Proceedings of the 2006 International Design and Engagability Conference.

[9] Nicole C Krämer. 2008. Soziale Wirkungen virtueller Helfer: Gestaltung und Evaluation von Mensch-Computer-Interaktion. W. Kohlhammer Verlag.

[10] Nicole C Krämer, Gale Lucas, Lea Schmitt, and Jonathan Gratch. 2018. Social Snacking with a Virtual Agent - On the Interrelation of Need to Belong and Effects of Social Responsiveness When Interacting with Artificial Entities. International Journal of Human-Computer Studies 109 (2018), 112-121.

[11] Chih-Chin Liang, Wen-Yau Liang, and Tzu-Lan Tseng. 2019. Evaluation of Intelligent Agents in Consumer-to-Business E-Commerce. Computer Standards \& Interfaces 65 (2019), 122-131.

[12] Masahiro Mori. 1970. Bukimi no tani [The Uncanny Valley]. Energy 7 (1970), 33-35.

[13] Susanne Schmidt, Gerd Bruder, and Frank Steinicke. 2019. Effects of Virtual Agent and Object Representation on Experiencing Exhibited Artifacts. Computers \& Graphics 83 (2019), 1-10.

[14] Nicole Sträfling, Ivonne Fleischer, Christin Polzer, Detlev Leutner, and Nicole C Krämer. 2010. Teaching Learning Strategies with a Pedagogical Agent. Fournal of Media Psychology (2010), 73-83.

[15] Carolin Straßmann and Nicole C Krämer. 2017. A Categorization of Virtual Agent Appearances and a Qualitative Study on Age-Related User Preferences. In Proceedings of the 2017 International Conference on Intelligent Virtual Agents. 413-422.

[16] Carolin Straßmann and Nicole C Krämer. 2018. A Two-Study Approach to Explore the Effect of User Characteristics on Users' Perception and Evaluation of a Virtual Assistant's Appearance. Multimodal Technologies and Interaction 2, 4 (2018), 66.

[17] Carolin Straßmann, Nicole C Krämer, Hendrik Buschmeier, and Stefan Kopp. 2020. Age-Related Differences in the Evaluation of a Virtual Health Agent's Appearance and Embodiment in a Health-Related Interaction: Experimental Lab Study. Journal of Medical Internet Research 22, 4 (2020), e13726.

[18] Araz Taeihagh and Hazel Si Min Lim. 2019. Governing Autonomous Vehicles: Emerging Responses for Safety, Liability, Privacy, Cybersecurity, and Industry Risks. Transport Reviews 39, 1 (2019), 103-128.

[19] Silke ter Stal, Marijke Broekhuis, Lex van Velsen, Hermie Hermens, and Monique Tabak. 2020. Embodied Conversational Agent Appearance for Health Assessment of Older Adults: Explorative Study. FMIR Human Factors 7, 3 (2020), e19987.

[20] Xiaohui Zhang, Shufeng Tang, Xinhua Liu, Reza Malekian, and Zhixiong Li. 2019. A Novel Multi-Agent-Based Collaborative Virtual Manufacturing Environment Integrated with Edge Computing Technique. Energies 12, 14 (2019), 2815. 